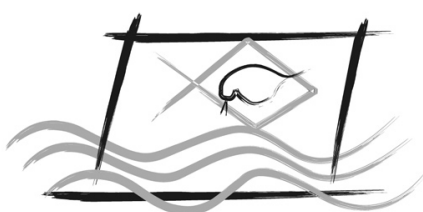

ECOTOX - BRASIL

\title{
Revisão dos ensaios crônicos de curta duração com embriões do mexilhão Perna perna (L.)
}

\author{
C.C. Del Cielo; J. Malgarin \& C. Resgalla JR.* \\ Universidade do Vale do Itajaí - UNIVALI - Centro de Ciências Tecnológicas da Terra e do Mar - CTTMar. \\ Rua Uruguai, 458, Itajaí, SC, 88.302-202
}

(Received November 08, 2012; Accept August 26, 2013)

\begin{abstract}
Review of embryo-larval assay of the mussel Perna perna (L.) - The objective of this work was to review the protocol of the mussel Perna perna embryo-larval test and suggest standardization of the induction technique, exposing time and effect observed in larvae at the end of the experiment. To meet the objectives were employed different physical and chemical methods to accelerate the release of gametes and the success of this release was compared to the index condition of the organisms. Ecotoxicological tests were also performed using the metals cadmium, lead, zinc, copper, chromium and the detergent sodium dodecyl sulfite (SDS) to assess the exposure time and morphological types of effects and their influence on estimates of Median Effective Concentration $\left(\mathrm{EC}_{50}\right)$. The most efficient induction method to stimulate the release of gametes was the continuous flow system of sea water, but it had a close relationship with the maturation of organisms. It was observed that the short term embryo-larval test can be executed in 24 hours since protracted larvae are considered normal as observed in control flasks experiments. Finally, embryo-larval test of the P. perna showed sensitivity in the same order of magnitude as compared to Lytechinus variegatus the main species of sea urchin commonly used in marine chronic tests in Brazil.
\end{abstract}

Key-words: Perna perna, Bivalvia, toxicity, condition index.

\section{INTRODUÇÃO}

No Brasil, o uso de embriões e larvas do mexilhão Perna perna (Linnaeus) começou tardiamente a ser investigado no final da década de 90 e início de 2000 e foi coincidentemente realizado por três grupos de pesquisa diferentes (Reis Fo., 1999; Zaroni, 2000, Zaroni et al., 2005; Reis Fo. \& Resgalla Jr., 2002 e Jorge, 2003). Em todos os casos, houve uma adaptação da norma estabelecida pela ASTM (1992) para o uso de Perna perna como organismo teste na avaliação do potencial tóxico de substâncias puras, amostras de água e da fração aquosa de sedimentos em ensaios crônicos de curta duração. Entretanto, o protocolo apresentado pela ASTM (1992) foi elaborado em base de quatro espécies de bivalves nativos de regiões temperadas, o que de certa forma não resumem a variabilidade observada no universo de ensaios com bivalves apresentado por His et al. (1999).
Somado a isto, as adaptações aos ensaios ignoraram o conhecimento gerado sobre a biologia da espécie, e que podem de maneira significativa alterar os métodos de execução dos experimentos (Resgalla Jr. \& Moraes, 2008). Os problemas metodológicos estão concentrados nas técnicas de obtenção de gametas, tempo do experimento e tipos morfológicos das larvas que caracterizam os efeitos adversos observados provocados por substâncias tóxicas. Além disto, a sensibilidade de embriões e larvas de $P$. perna ainda não é totalmente conhecida, necessitando comparações com os experimentos realizados com outros ensaios crônicos marinhos.

Para $P$. perna, não existe uma técnica rápida e segura para a liberação de gametas. A falta de uma técnica padrão muitas vezes limita o seu uso como ensaio de rotina em muitos laboratórios. Na literatura existem várias referências sobre as técnicas de indução (Iwata, 1950; Sagiura, 1962; Loosanoff \& Davis, 1963; Bayne, 1976; Zaroni, 2000), mas o grau de

*Corresponding author: Charrid Resgalla Jr.; e-mail: cresgalla@univali.br 
maturação dos organismos é frequentemente citado como uma condicionante ao sucesso da liberação de gametas.

Quando inicialmente proposto o protocolo de ensaio com P. perna, a adaptação da ASTM (1992) baseada para Mytilus edulis recomenda o tempo de teste de 48 horas. Entretanto, a biologia do desenvolvimento de $P$. perna é mais rápida e ocorre em temperaturas mais elevadas, atingindo a larva D em 24 horas (Romero, 1980), ou seja, em metade do tempo da espécie do hemisfério norte.

Por fim, problemas relacionados à identificação dos tipos morfológicos característicos de efeitos adversos a serem observados e considerados no final do experimento após a exposição das larvas aos tóxicos são frequentes. Vários autores consideram a forma da larva D protraída como efeito de ação deletéria. Entretanto este fato foi destacado por Romero (1980) como uma característica comum da fase de desenvolvimento do P. perna.

Estas questões levam à indicação de que o protocolo de execução dos ensaios com $P$. perna devem ser investigados a luz dos conhecimentos gerados objetivando principalmente o seu uso como alternativa para ensaios crônicos marinhos.

\section{MATERIAL E MÉTODOS}

Organismos adultos do mexilhão Perna perna (tamanho entre 7 a $8 \mathrm{~cm}$ ) foram coletados no município de Penha, litoral norte de Santa Catarina (Brasil) (26 $48^{\circ} \mathrm{S} 48^{\circ} 36^{\prime} \mathrm{W}$ ) e transportados a seco até o Laboratório de Ecotoxicologia do CTTMAr (UNIVALI), onde foram imediatamente disponibilizados para a indução de liberação de gametas.

Logo que os organismos chegavam ao laboratório, foram removidas as incrustações superficiais com o auxílio de uma espátula e o bisso foi retirado com uma tesoura. Em seguida, os organismos passavam por uma lavagem com água doce e foram transferidos para uma bandeja contendo água da torneira e hipoclorito de sódio comercial (diluição de 1:1000) durante 30 minutos para profilaxia externa.

\section{Métodos de estímulo à liberação de gametas}

Foram testados as seguintes técnicas de indução de liberação de gametas:

1. Castigo - os mexilhões ficaram expostos ao ar por 30 minutos e depois foram submersos em água do mar (Bayne, 1976, Loosanoff \& Davis, 1963);

2. Choque térmico - os organismos foram mantidos em água do mar com temperatura de $24^{\circ} \mathrm{C}$ e posteriormente foram transferidos para uma bandeja com água do mar à $35^{\circ} \mathrm{C}$ (Bayne, 1976, Loosanoff \& Davis, 1963);

3. Indução elétrica - com estímulos de choque elétrico de 30 volts e 1 ampère por 5 segundos em cada organismo na região do músculo anterior e do bisso, tendo um intervalo de 30 minutos entre cada choque e com um segundo estímulo elétrico (Iwata, 1950; Sagiura, 1962);
4. Extrusão de Gônadas - foram realizados cortes de parte das gônadas e posterior lavagem com água do mar para a obtenção de ovócitos e espermatozóides. Esse material foi posteriormente analisado em microscópio;

5. Sistema de fluxo contínuo - organismos submetidos ao fluxo de água do mar filtrada em sistema de circulação fechada;

6. Utilização de substâncias químicas - injeção de substâncias químicas na cavidade do manto com auxílio de agulha cirúrgica e posterior agitação dos animais para homogeneização interna. Depois da injeção os organismos foram transferidos para bandeja plástica contendo água do mar. As substâncias utilizadas foram:

a - Cloreto de potássio $(\mathrm{KCl})$ a $5 \mathrm{mM}(0,37 \mathrm{~g}$ de $\mathrm{KCL}$ em 1000 mL) (Reis Fo \& Resgalla Jr., 2002; Sagara, 1958);

b - Adrenalina $1 \mathrm{~g} \mathrm{~mL}^{-1}$ seguindo-se de 4 injeções com intervalos de 30 minutos;

c - Cloreto de Amônio $\left(\mathrm{NH}_{4} \mathrm{Cl}, 2 \mathrm{~g} \mathrm{~L}^{-1}\right)$ seguindo-se de 4 injeções de $1 \mathrm{~mL}$ com intervalos de 30 minutos (Reis Fo \& Resgalla Jr., 2002);

$\mathrm{d}$ - Verapamil $1 \mathrm{~g} \mathrm{~L}^{-1}$ (inibidor de canal de cálcio) seguindose de 4 injeções com intervalos de 1 hora;

e - Glicose $1 \mathrm{~g} \mathrm{~L}^{-1}$ (açúcar) seguindo-se de 3 injeções de 1 $\mathrm{mL}$ com intervalos de 1 hora;

$\mathrm{f}$ - Analgésico Lidocaína 2,050 mg L $\mathrm{m}^{-1}$ e Epinefrina 1,001 $\mathrm{mg} \mathrm{L}^{-1}$ com 3 injeções em intervalos de 30 minutos;

$\mathrm{g}$ - Estradiol $4 \mathrm{mg} \mathrm{L}^{-1}, 1 \mathrm{mg} \mathrm{L}^{-1}$ e $0,5 \mathrm{mg} \mathrm{L}^{-1}$ (hormônio sexual) com 3 injeções em intervalos de 1 hora.

\section{Índice de condição}

Após a realização das induções e da liberação dos gametas (quando positivos) foi obtida a biometria de 30 mexilhões, com medição de comprimento total, volume de concha fechada e aberta. As partes moles foram separadas das conchas e secas em estufa a $60^{\circ} \mathrm{C}$ por 48 horas, para a obtenção de seu peso seco. O índice de condição foi estimado segundo a equação proposta por Walne (1970):

$I C=\frac{P s}{V c h} \times 1000$

Sendo:

$$
\begin{aligned}
& \text { IC = Índice de condição } \\
& \text { Ps = Peso seco da parte mole do mexilhão (mg) } \\
& \text { Vch = Volume interno da concha }(\mathrm{mL})
\end{aligned}
$$

\section{Ensaios ecotoxicológicos}

Comaliberação de gametas porumas das técnicas anteriores, os organismos foram transferidos para placas de Petri com água do mar para a coleta de óvulos e espermatozoides. Os óvulos foram concentrados em béqueres com água do mar e os espermatozoides foram concentrados em béqueres a seco 
sobre gelo. Para a fecundação adicionaram-se algumas gotas de solução espermática $(0,5 \mathrm{~mL}$ de espermatozoides em 10 $\mathrm{mL}$ de água do mar) na solução de óvulos e observação da formação do corpúsculo polar (Reis Fo. \& Resgalla Jr. 2002). Os ensaios de toxicidade utilizando $\mathrm{Cd}, \mathrm{Pb}$ (ambos a partir de soluções padrões de absorção atômica $\left.\mathrm{Merck}^{\circledR}\right)$, sulfato de zinco $\left(\mathrm{ZnSO}_{4} \cdot 7 \mathrm{H}_{2} \mathrm{O}\right)$, sulfato de cobre $\left(\mathrm{CuSO}_{4} \cdot 5 \mathrm{H}_{2} \mathrm{O}\right)$, dicromato de potássio $\left(\mathrm{K}_{2} \mathrm{Cr}_{2} \mathrm{O}_{7}\right)$ e dodecil sulfato de sódio (DSS) foram realizados em 6 concentrações, com 4 réplicas para cada concentração e 8 frascos controle com água do mar. Em cada frasco foram adicionados $200 \mu \mathrm{L}$ de solução de ovos (média de 400 ovos) em $10 \mathrm{~mL}$ da solução teste com sua incubação à $25^{\circ} \mathrm{C}$ (Reis Fo. \& Resgalla Jr. 2002). Os ensaios foram realizados em duplicata para a finalização em 24 horas e em 48 horas. Para a finalização foi adicionado $1 \mathrm{~mL}$ de formol a $4 \%$ em cada frasco teste. Foram realizados um total de 11 ensaios com $\mathrm{Pb}, 7$ com $\mathrm{Cd}$ e 4 para $\mathrm{Zn}, \mathrm{Cu}, \mathrm{Cr}$ e DSS.

\section{Análise dos resultados}

A análise dos frascos teste constou na contagem das larvas em câmara de contagem de Sedwick-Rafter, contendo $1 \mathrm{~mL}$ da solução teste com os organismos fixados. Foram quantificados os 100 primeiros organismos classificando-os em Larva D (normal), Larva D Protraída e Anormal (Fig. 1) (His \& Beiras, 1995; Reis Fo \& Resgalla Jr., 2002). A partir desta quantificação

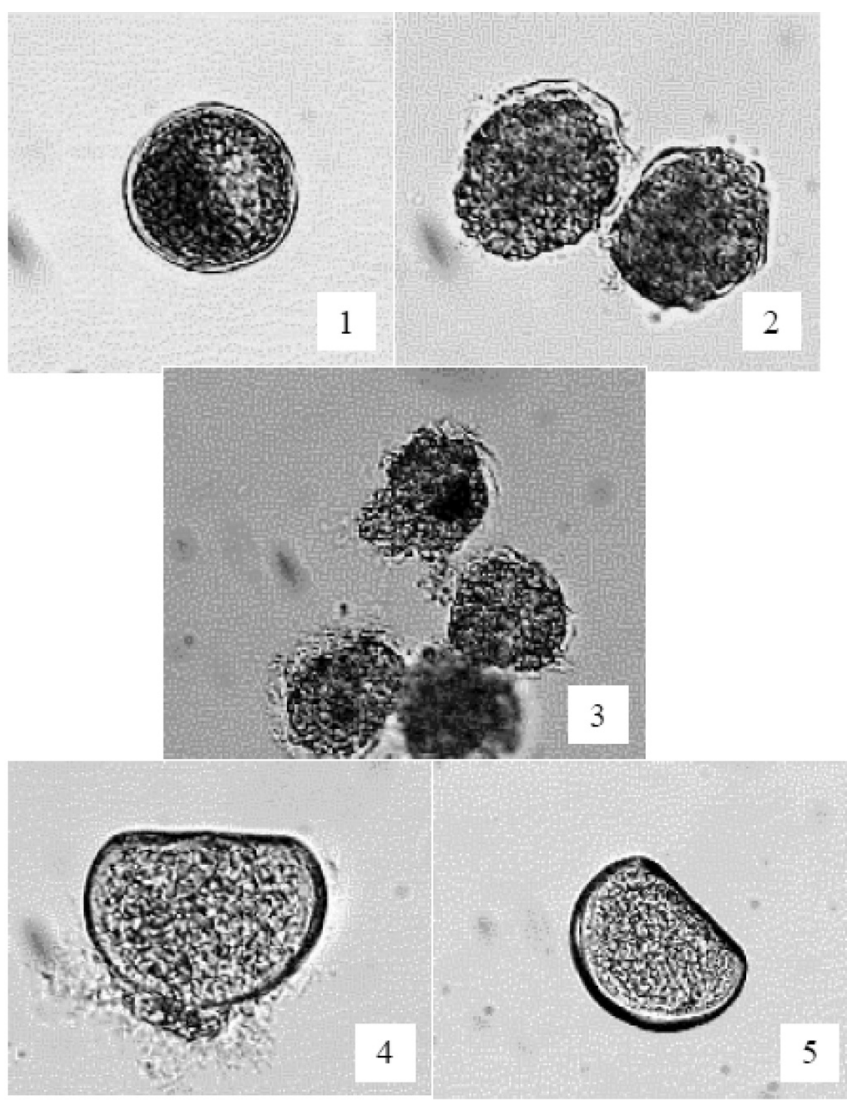

Figura 1 - Estágios de desenvolvimento do mexilhão Perna perna no encerramento dos ensaios ecotoxicológicos: 1: Ovo, 2: Trocóforas, 3:

Trocófora em decomposição (efeito), 4: D protraída e 5: D normal. Larvas 4 e 5 com 48 horas de idade. Retirado de Reis Fo. \& Resgalla Jr. (2002). foram estimados os percentuais de efeito, considerando ora as larvas D protraídas como efeito e ora como normais para avaliação da influência deste critério de classificação nas estimativas da $\mathrm{CE}_{50}$ (Concentração Efetiva Mediana). O critério de validação adotado para os ensaios foi de um máximo de $30 \%$ de efeito nos frascos controle quando as larvas protraídas foram classificadas como anormais em exposição de 24 horas e de $20 \%$ para os demais ensaios. $\mathrm{A} \mathrm{CE}_{50}$ foi estimada pelo método de Spearman-Karber (USEPA, 2002).

\section{Tratamento dos dados}

As comparações entre os valores de $\mathrm{CE}_{50}$ obtidos em diferentes tempos de exposição ( 24 e 48 horas) e diferentes critérios de classificação das larvas (anormais, protraídas e larvas D) de $P$. perna ao final do experimento foram realizadas por Análise de Variância (ANOVA) com um nível de significância de $5 \%$.

\section{RESULTADOS}

\section{Indução a desova}

Das técnicas de estímulo à liberação de gametas, somente os métodos de fluxo contínuo, castigo e choque térmico (Tabela 1) geraram ovócitos viáveis para uso posterior em ensaios ecotoxicológicos. A técnica do fluxo contínuo foi a mais eficaz, com uma média de liberação de $16,5 \%$ dos organismos induzidos.

As técnicas do castigo e de choque térmico resultaram em liberação de gametas de forma esporádica, mas normalmente em quantidade insuficiente para a realização dos ensaios ecotoxicológicos. O método de indução elétrica apresentou 9,3\% dos casos de liberação, que apesar de ter sido a segunda técnica mais eficiente, não disponibilizou material de qualidade para a fecundação.

O uso de estimuladores químicos não apresentou liberações de gametas, não mostrado, portanto, técnicas confiáveis de

Tabela 1 - Sumário dos métodos empregados para extrusão de gametas em Perna perna e a viabilidade de uso em ensaios ecotoxicológicos.

\begin{tabular}{|c|c|c|c|c|}
\hline Método & $\begin{array}{l}\mathrm{N}^{\mathrm{o}} \mathrm{de} \\
\text { testes }\end{array}$ & $\begin{array}{c}\mathrm{N}^{\circ} \text { médio de } \\
\text { organismos } \\
\text { por teste }\end{array}$ & $\begin{array}{c}\text { Percentagem } \\
\text { média de } \\
\text { liberação }\end{array}$ & $\begin{array}{l}\text { Viabilidade } \\
\text { para testes de } \\
\text { toxicidade }\end{array}$ \\
\hline Fluxo contínuo & 27 & 30 & $16,5 \%$ & + \\
\hline Castigo & 5 & 30 & $5,3 \%$ & + \\
\hline Choque térmico & 5 & 40 & $2,8 \%$ & + \\
\hline Indução elétrica & 5 & 37 & $9,3 \%$ & - \\
\hline $\mathrm{KCl}$ & 3 & 83 & $2,7 \%$ & - \\
\hline Adrenalina & 3 & 100 & $6,9 \%$ & - \\
\hline Verapamil & 3 & 50 & $2 \%$ & - \\
\hline Glucose & 2 & 100 & $4 \%$ & - \\
\hline Estradiol $\left(0,5 \mathrm{mg} \mathrm{L}^{-1}\right)$ & 3 & 50 & $0 \%$ & - \\
\hline Estradiol $\left(1 \mathrm{mg} \mathrm{L}^{-1}\right)$ & 3 & 50 & $0 \%$ & - \\
\hline Estradiol $\left(4 \mathrm{mg} \mathrm{L}^{-1}\right)$ & 3 & 100 & $0 \%$ & - \\
\hline $\mathrm{NH}_{4} \mathrm{Cl}$ & 2 & 50 & $0 \%$ & - \\
\hline Extrusão de gônadas & 3 & 100 & $0 \%$ & - \\
\hline Analgésico & 3 & 50 & $0 \%$ & - \\
\hline
\end{tabular}


uso independente da época do ano e do estado de maturação dos organismos.

\section{Índice de condição}

A avaliação do índice de condição foi realizada em relação à técnica de fluxo contínuo devido ao maior sucesso de liberação de gametas viáveis (Fig. 2). Foi observada uma alta concordância entre estes dois parâmetros e com sazonalidade marcante com picos na primavera/verão e no final do outono.

\section{Testes de toxicidade}

O desenvolvimento normal das larvas de P. perna indicou que as larvas do tipo protraídas são abundantes nas primeiras 24 horas de ensaio (Fig. 3) podendo apresentar 14,46\% das larvas. Em 48 horas de ensaio, o percentual de larvas protraídas caiu para menos de 5\% indicando que esta seria uma fase normal de desenvolvimento destes organismos.

Comparando-se os resultados dos testes de toxicidade realizados com os metais $(\mathrm{Pb}, \mathrm{Cd}, \mathrm{Zn}, \mathrm{Cr}, \mathrm{Cu})$ e o detergente DSS, nos tempos de exposição de 24 e 48 horas, observouse que os valores de $\mathrm{CE}_{50}$ estão dentro do mesmo intervalo de variação (Fig. 4), tanto em termos de tempo de exposição como em termos de critérios adotados na classificação de larvas com e sem efeitos, não apresentando diferenças significativas entre os valores de $\mathrm{CE}_{50}$ (Tabela 2). Entretanto, devido à alta porcentagem de larvas $\mathrm{D}$ protraídas observadas nos frascos controle em 24 horas de exposição, a validação do experimento só é possível com percentuais de efeito de até $30 \%$.

\section{DISCUSSÃO}

A adequação de ensaios crônicos de curta duração com o mexilhão Perna perna vai além das práticas de padronização de seu protocolo de execução, mas visa principalmente a preservação das espécies de ouriço normalmente utilizadas como organismos teste para este tipo de ensaio. $\mathrm{O}$ estimulo do uso de espécies cultiváveis ou de interesse econômico na

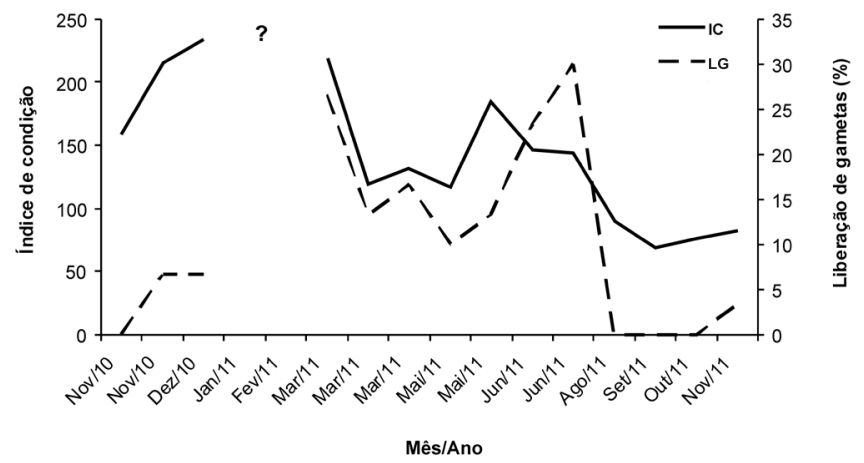

Figura 2 - Variação temporal do índice de condição e da porcentagem de liberação de gametas por fluxo contínuo ao longo dos anos de 2010 a 2011.

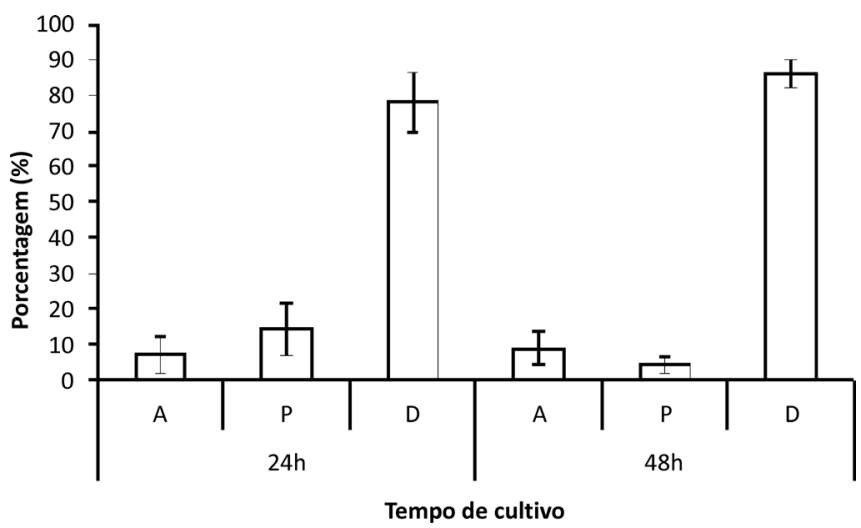

Figura 3 - Porcentagem média (barra) e desvio padrão (linha) de larvas anormais (A), protraídas (P) e larvas D (D) por tempo de cultivo observadas nos frasco controle para os ensaios embrio-larval de Perna perna.
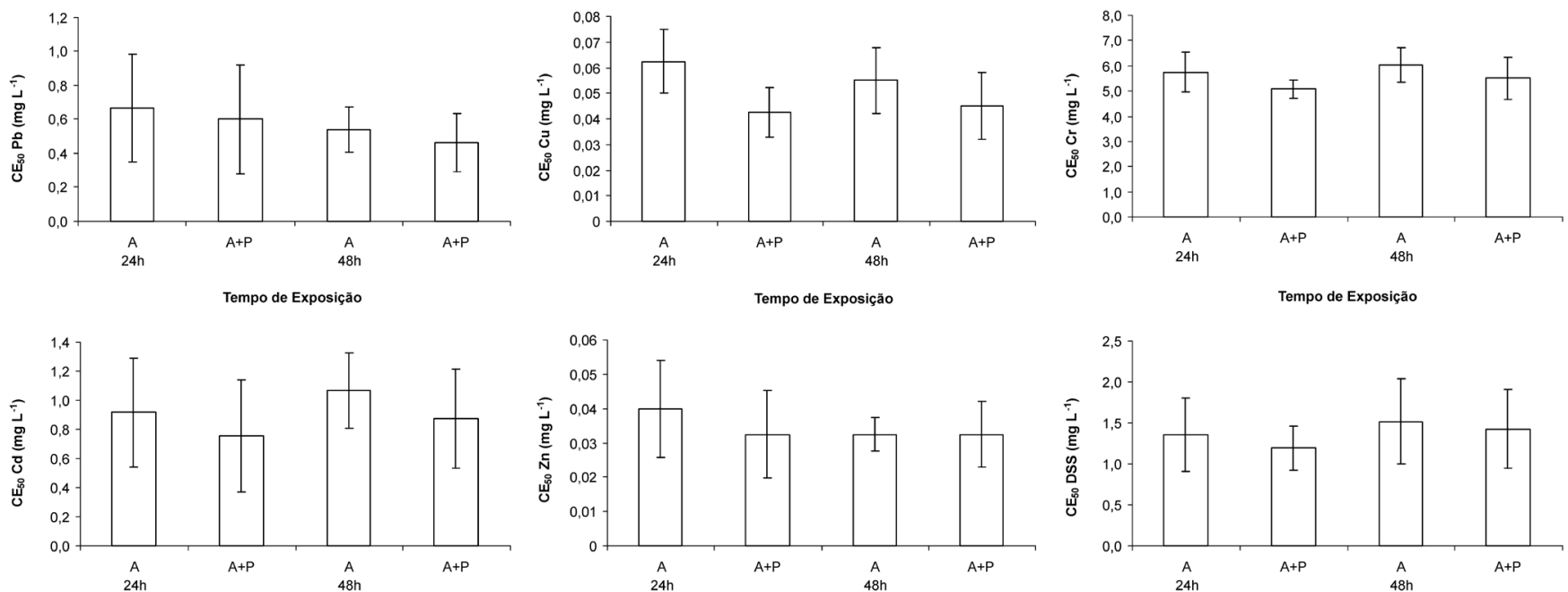

Figura 4 - Valores médios de $\mathrm{CE}_{50}$ (barra) e desvio padrão (linha) para $\mathrm{Pb}, \mathrm{Cd}, \mathrm{Cu}, \mathrm{Zn}$, Cr e $\mathrm{DSS}$ em dois tempos de exposição (24 e 48 horas) e adotandose dois critérios de classificação de efeitos das larvas (anormais ou atrasadas - A e anormais somada a protraídas $-\mathrm{A}+\mathrm{P}$ ) para ensaios embrio-larval de $P$. perna. Para os ensaios com $\mathrm{Pb}$ os dados representam médias de 11 ensaios, 7 para Cd e 4 para $\mathrm{Cu}, \mathrm{Zn}, \mathrm{Cr}$ e DSS. 
Tabela 2 - Resultados da ANOVA entre os valores de $\mathrm{CE}_{50}$ para diferentes tempo de exposição e efeitos observados em ensaios com lavras de $P$. perna sob efeito de chumbo, cádmio, cobre, zinco, cromo e dodecil sulfato de sódio, para um nível de significância de 5\%.

\begin{tabular}{lccc}
\hline Tóxico & F crítico & F calculado & Graus de liberdade \\
\hline $\mathrm{Pb}$ & 2,92 & 1,220 & 3,30 \\
$\mathrm{Cd}$ & 3,10 & 0,792 & 3,20 \\
$\mathrm{Cu}$ & 3,49 & 2,216 & 3,12 \\
$\mathrm{Zn}$ & 3,49 & 0,433 & 3,12 \\
$\mathrm{Cr}$ & 3,49 & 1,521 & 3,12 \\
$\mathrm{DSS}$ & 3,49 & 0,340 & 3,12 \\
\hline
\end{tabular}

aquicultura podem oferecer vantagens ecológicas e agregar valor aos testes de toxicidade.

O mexilhão Perna perna apresenta uma longa história de conhecimento biológico no Brasil mas igualmente disperso e só tardiamente compilado o que vem impedindo o seu uso em diferentes áreas de avaliação de impacto (Resgalla Jr. et al., 2008). Os estudos sobre o ciclo de vida da espécie vêm sendo realizados desde as décadas de 50 e 60 no Brasil e apresentaram grande interesse nas décadas de 70 e 80 devido ao desenvolvimento da mitilicultura. Graças a este conhecimento gerado sabe-se que $P$. perna apresenta picos de desova na primavera/verão e outono tanto na costa sudeste de São Paulo (Lunetta, 1969) como em Santa Catarina (Marenzi \& Branco, 2005; Gomes, 1999).

Para os Mytilidae estudados fora do Brasil é consenso de que o principal fator controlador de sua desova é atribuída as variações de temperatura (Baird, 1966; Rojas, 1971; Andreu, 1976; Acuña, 1977; Kent, 1979; Morales, 1983). Durante a primavera são dois os fatores que influenciam a desova dos mexilhões, o aumento na temperatura e ao aumento da quantidade de alimento disponível, principalmente de origem fitoplanctônica (Camacho, 1991).

Como indicativo deste período de desova o uso do índice de condição reflete diretamente o estágio de desenvolvimento das gônadas dos organismos. Este índice indica qual a melhor época de reprodução do animal, bem como a de colheita e comercialização, uma vez que quanto maior é o valor do índice de condição, maior é o peso do organismo (Gomes, 1999). Segundo Lunneta (1969), o índice de condição também pode ajudar a controlar as coletas de sementes do mexilhão, fazendo com que esta atividade não prejudique os estoques naturais. Na parte biológica, este índice também indica o ciclo sexual, pois as gônadas ocupam a região do manto, e isso representa uma alta proporção do total das partes moles (Marenzi, 1992).

Os resultados obtidos indicam que existe uma forte relação entre o índice de condição e o sucesso de liberação de gametas e um período de menores chances de obtenção do material biológico no inverno. Esta sazonalidade do índice de condição para $P$. perna também já foi destacada por Marques \& Pereira (1998), Peres (1981) e Gomes (1999). Por outro lado, as maiores liberações de gametas sempre ocorreram no final dos períodos de engorda dos organismos, ou no fim da primavera, verão e outono.
Com isso nota-se que o sucesso de liberação ou obtenção de gametas está mais intimamente ligado à maturação dos organismos do que a alguma técnica de estímulo mais efetiva. Este fato explica de certa forma a falta de sucesso na grande maioria das técnicas aplicadas, neste trabalho, para a indução de desova. A técnica de fluxo continuo de água do mar pode ser a técnica mais próxima do uso de oxidantes (peróxido de hidrogênio) que estimularia a síntese hormonal pela oxigenação da água circundante (Morse et al., 1978).

Das demais técnicas, a mais popular é a indução térmica frequentemente citada para a obtenção de larvais de bivalves (Loosanoff \& Davis, 1963; His \& Beiras, 1995), mas que normalmente geram pouco material para uso em ensaios. A extrusão por meio da raspagem das gônadas, que também foi utilizada por Brereton et al. (1973), apresenta problemas em disponibilizar material gâmico que ainda não estejam prontos para fecundação. Por isso é necessária a avaliação microscópica do estado morfológico dos ovócitos, como foi sugerido por Zaroni (2000). Esta técnica de raspagem foi utilizada por Vélez \& Martinez (1967), Carvalhaes (2001) e Gomes (1986) como técnicas eficientes para utilização posterior dos gametas.

Ainda que muito citadas na literatura (Sagara, 1958), as técnicas de injeção de compostos químicos não mostraram um resultado positivo para liberação de gametas. Uma alternativa para aumentar as chances de obtenção de gametas seria a combinação de métodos associado ao uso de um grande número $(>100)$ de organismos a serem induzidos.

\section{Cultivo das larvas e testes de toxicidade}

Segundo His et al. (1999) existe uma relação entre o tempo de exposição de um experimento embrio-larval de bivalves e o percentual de efeito observado nos frascos controle. De qualquer forma, esta relação também é dependente dos critérios adotados na classificação das larvas e nos tipos morfológicos que possam caracterizar os efeitos adversos dos tóxicos avaliados.

Desta forma, observou-se neste trabalho que existe a necessidade de um período de 48 horas para que as larvas de $P$. perna atinjam um percentual mínimo de $20 \%$ de larvas normais em condições ideais de cultivo (controle). Por outro lado, as larvas D protraídas, quando consideradas normais, este percentual é atingido em 24 horas de cultivo, o que foi igualmente observado por Reis Fo (1999) e Reis Fo. \& Resgalla Jr. (2002). Larvas D protraídas são caracterizadas por lavas que apresentam um manto maior que a protoconcha, característica necessária para a sua natação (Romero, 1980). Com a idade de 48 horas, as larvas D, agora umbuladas, apresentam uma concha maior que o manto, tendo a sua natação reduzida e concentrando-se, por isto, no fundo dos frascos teste.

Comparando-se com as recomendação apresentadas pela ASTM (1992) e por His et al. (1999), o correto tempo de exposição deste tipo de ensaio é de 24 horas quando considerado o estágio de desenvolvimento adotado como critério de encerramento do experimento (similar ao que 
acontece com os ensaios embrio-larval de ouriços). Entretanto, comparando-se os valores de sensibilidade para as diferentes substâncias tóxicas testadas, este ensaio pode ser realizado tanto em 24 como em 48 horas, ressaltando-se os tipos morfológicos das larvas a serem adotadas como critérios de efeito e não efeito tóxico e dos percentuais de efeito observado nos frascos controle. Somado a isto, os ensaios com 48 horas de duração podem apresentar maiores riscos de invalidação por mortalidade frequentemente observada nas larvas nos estágios mais avançados (e.g. larvas umbuladas). Por outro lado, o tempo de ensaio pode também ser dependente pela temperatura dos experimentos, podendo ser executados a 20 e $25{ }^{\circ} \mathrm{C}$ (Zaroni et al., 2005) mas sem interferência nos resultados de sensibilidade como observado por Reis Fo. \& Resgalla Jr. (2002) em testes sob efeito do DSS em diferentes tempos de exposição (24 e 48 horas).

\section{Sensibilidade}

Com o objetivo de utilizar os ensaios crônicos de curta duração embrio-larval de $P$. perna em substituição aos ensaios com ouriço-do-mar, a comparação dos valores de $\mathrm{CE}_{50}$ com a espécie de Echinodermata mais utilizada em ensaios embrio-larval no Brasil indicou, com exceção do $\mathrm{Cd}$, uma sensibilidade na mesma ordem de grandeza (Tabela 3 ). Resultado semelhante foi observado por Zaroni et al. (2005) para o detergente DSS.

Estes resultados sugerem que $P$. perna poderia ser utilizado como uma alternativa aos ensaios com ouriços-do-mar, apresentando a vantagem de ser uma espécie cultivada e de não apresentar impactos por sua retirada dos estoques naturais.

\section{CONCLUSÕES}

O método mais eficaz para liberação de gametas de Perna perna para utilização em ensaios ecotoxicológicos foi $\mathrm{o}$ de fluxo contínuo de água do mar, entretanto o sucesso da liberação de gametas está intimamente relacionada com a maturação dos organismos, que apresenta uma sazonalidade conferindo uma desvantagem na sua utilização.

Os testes de toxicidade crônicos com o mexilhão Perna perna podem apresentar duração de 24 horas onde as larvas protraídas podem ser consideradas normais para percentuais de efeito aceitáveis de $20 \%$ nos frasco controle ou podem ser consideradas anormais para percentuais de efeito aceitável de $30 \%$ nos frascos controle.

De forma geral a sensibilidade de embriões e larvas de $P$. perna é comparável com a espécie de ouriço-do-mar normalmente utilizada nos ensaios crônicos de curta duração no Brasil.

\section{REFERÊNCIAS}

ACUÑA C., A. 1977. Crescimiento y índice de engorda del mejillón Perna perna (L.) cultivado en el Golfo de Cariaco, Venezuela. FAO Fish. Report. Roma, 200:1-9.

ANDREU, B. 1976. El cultivo del mejillón en Europa. An. Acad. Bras. Ciênc. 45: 22-36.

ASTM, American Society of Testing and Materials. 1992. ASTM E 724-89 Standart guide for conduction static toxicity tests starting with embryos of four species of saltwater mollusks. In: ASTM - American Society for Testing and Materials. Annual Book of ASTM Standards: water and environmental technology. Philadelphia. 11:4:377-394.

BAIRD, R.H. 1966. Factors affecting the growth and condition of mussels (Mytilus edulis L.). Fisheries and Food Fishery Investigations Series, 2, $52 \mathrm{p}$.

BAYNE, B.L. 1976. Marine mussel: Their ecology and physiology. Londres: Cambridge University Press, 504p.

BRERETON, A.; LORD, H.; THORTON, I. \& WEBB, J.S. 1973. Effect of zinc on growth and development of larvae of the pacific oyster Crassostrea gigas. Mar. Biol. 19:96-101. http://dx.doi. org/10.1007/BF00353580

CAMACHO, A.P. 1991. Cultivo del mejillón en la batea. In: CASASBELLAS, M.A.C., CAMACHO, A.P. \& FERNÁNDEZ (eds.) I. Unidades didácticas de acuicultura. Alva, 12, 53 p.

CARVALHAES, A.L. 2001. Método de extrusão: alternativa para testes de toxicidade com o mexilhão Perna perna (Linné, 1758). Dissertação (Mestrado em Biologia Marinha) - Universidade Federal Fluminense, Rio de Janeiro, 70p.

GOMES, L.A.O. 1986. Cultivo de Crustáceos e Moluscos. São Paulo: Nobel. 226 p.

GOMES, R.O.M. 1999. Determinação dos fatores ambientais atuantes na variação do índice de condição do mexilhão Perna perna (Linné, 1758) em cultivo na enseada da Armação do Itapocoroy, Penha, SC. Monografia do curso de Oceanografia, UNIVALI, 38p.

HIS, E. \& BEIRAS, R. 1995. Monitoring fresh and brackish water quality around shellfish farming areas with a bivalve embryo and larva simplified Biossay method. Oceanologica Acta. 18:591595.

HIS, E., BEIRAS, R. \& SEAMAN, M.N.L. 1999. The Assessment of Marine Pollution - Bioassays with Bivalve Embryos and Larvae. Advances Mar. Biol. 37:1-178. http://dx.doi.org/10.1016/S00652881(08)60428-9

IWATA, K.S. 1950. Spawning of Mytilus edulis: discharge by electrical stimulation. Bull. Japanese Soc. Sc. Fish. 15:443-446.

JORGE, R.A.V.C. 2003. Avaliação de impacto ambiental sobre o ecossistema marinho utilizando larvas de mexilhões (Perna perna) (Linnaeus, 1758) (Mollusca: Bivlavia) como bioindicadores, através de técnicas ecotoxicológicas. Tese de Doutorado. Escola de Engenharia de São Carlos, USP. 505p.

KENT, R.M.L. 1979. The influence of heavy infestations of

Tabela 3 - Intervalo dos valores de $\mathrm{CE}_{50}\left(\mathrm{mg} \mathrm{L}^{-1}\right)$ para $\mathrm{Cd}, \mathrm{Pb}, \mathrm{Cu}, \mathrm{Zn}, \mathrm{Cr}$ e DSS em ensaios crônicos embrio-larval com o ouriço Lytechinus variegatus e o mexilhão Perna perna.

\begin{tabular}{|c|c|c|c|c|c|c|c|}
\hline Espécie & $\mathrm{Cd}$ & $\mathrm{Pb}$ & $\mathrm{Cu}$ & $\mathrm{Zn}$ & $\mathrm{Cr}$ & DSS & Autor \\
\hline $\begin{array}{l}\text { Lytechinus } \\
\text { variegatus }\end{array}$ & $4,21-5,89$ & $0,06-0,18$ & $0,008-0,03$ & $0,02-0,09^{*}$ & $2,30-2,60$ & $1,10-1,70$ & Soares (2011) \\
\hline Perna perna & $0,84-0,97$ & $0,50-0,63$ & $0,03-0,08$ & $0,02-0,06$ & $5,01-7,02$ & $0,98-2,03$ & Este trabalho \\
\hline
\end{tabular}

"segundo carta controle de sensibilidade do Letox/CTTMar. 
Polydora ciliata on the flesh content of Mytilus edulis. J. Mar. Biol. Assoc. U.K. 59(2):289-297. http://dx.doi.org/10.1017/ S0025315400042594

LOOSANOFF, V. L. \& DAVIS, H. 1963. Rearing of bivalve mollusks. Adv. Mar. Biol. 1:1-136. http://dx.doi.org/10.1016/ S0065-2881(08)60257-6

LUNETTA, J.E. 1969. Fisiologia da reprodução dos mexilhões (Mytilus perna - Mollusca Lamellibranchia). Bol. Zool. Biol. Mar. 26:33-111.

MARENZI, A.W.C. 1992. Aspectos biológicos e econômicos do cultivo de mexilhões Perna perna (Linné, 1758) (Molluscabivalvia), no litoral centro-norte catarinense. 1992. $135 \mathrm{f}$. Dissertação (Mestrado em Ciências Biológicas) - Universidade Federal do Paraná, Curitiba. 135p.

MARENZI, A.W.C. \& BRANCO, J.O.O. 2005. Mexilhão Perna perna (Linnaeus) (Bivalvia, Mytilidae) em cultivo na Armação do Itapocoroy, Santa Catarina, Brasil. Rev. Bras. Zool. 22(2):394399.

MARQUES, H. L. A. \& PEREIRA, R.T.L. 1998. Mexilhões: Biologia e Criação. Boletim técnico. São Paulo: Coordenadoria de Pesquisa Agropecuária. 32p.

MORALES, J.C. 1983. Acuicultura Marina Animal. Madri: MundiPrensa. 423 p.

MORSE, D.E., HOOKER, N. \& MORSE, A. 1978. Chemical control of reproduction in bivalve and gastropod molluscs. III: An inexpensive technique for mariculture of many species. Proceedings of the annual meeting - World Mariculture Soc. 9(1-4):543-547. http://dx.doi.org/10.1111/j.1749-7345.1978. tb00272.x

PERES, S. 1981. Estudo do ciclo reprodutivo de Pecten ziczac (Linné, 1758) (Mollusca: Bivalvia). Tese (Mestrado em Fisiologia) Instituto de Biociências da USP, São Paulo. 124p.

REIS Fo, R.W. 1999. Estudos preliminares envolvendo testes de toxicidade embrio-larvais com o mexilhão Perna perna (Linnaeus, 1758). Monografia curso de Oceanografia, UNIVALI, 37p.

REIS Fo, R.W. \& RESGALLA JR. C. 2002. Testes de toxicidade com embriões de Perna perna (Linnaeus, 1758) (Mollusca: Mytilidae): avaliações de indução e sensibilidade. Notas Téc. FACIMAR. 6:7-17.

RESGALLA Jr., C.; WEBER, L.I. \& CONCEIÇÃO, M.B. 2008. O mexilhão Perna perna: Biologia, ecologia e aplicações. Editora
Interciência, 319pp.

RESGALLA Jr. C. \& MORAES, R.B.C. 2008. Capítulo 15 - Uso de Perna perna em ensaios ecotoxicológicos, In: RESGALLA JR, C.; WEBER, L. I.; CONCEIÇÃO, M. B. (eds.), O mexilhão Perna perna: Biologia, ecologia e aplicações. Editora Interciência, p. 255-269.

ROJAS, A.V. 1971. Fluctuacion mensual del índice de engorde del mejilon Perna perna natural y cultivado. Bol. Inst. Oeanogr. Univ. Oriente. 10(2):3-8.

ROMERO, S.M.B. 1980. Características comportamentais e morfológicas dos estágios larvais de Perna perna (Lamellibranchia: Mytilidae) obtidos em laboratório. Bol. Fisiol. Animal. 4: 45-52.

SAGARA, J. 1958. Artificial discharge of reproductive elements of certain bivalves caused by trestment of sea water and by injection with $\mathrm{NH}_{4} \mathrm{OH}$. Bull. Japonese Soc. Sc. Fish. 23:505-510.

SAGIURA, Y. 1962. Electrical induction of spawning in two marine invertebrates (Urechis unicinctus, hermaphrodite Mytilus edulis). Biol. Bull. 123:203-206.

SOARES, J.B. 2011. Alternativas para os ensaios crônicos com Echinodermatas. Trabalho de Conclusão do Curso de Biologia, CTTMar/UNIVALI. 50pp.

USEPA, U.S. Environmental Protection Agency. 2002. Methods for measuring the acute toxicity of effluents and receiving waters to freshwater and marine organisms. 5 Ed. Washington. 266p.

VÉLEZ, R.A. \& MARTINEZ, E.R. 1967. Reproducción y desarollo larval experimental del mejillón comestible en Venezuela (Linnaeus, 1758). Bol. Inst. Orient. 6:266-285.

WALNE, P.R. 1970. The seasonal variation of meat and glycogen content of seven populations of oysters Ostrea edulis (L.) and a review of the literature. Min. Agric. Fish. Food. Fish. Invest. 26(2):1-33.

ZARONI, L.P. 2000. Testes de toxicidade com a utilização de embriões do mexilhão Perna perna (Linnaeus, 1758): Uma adequação do método. Dissertação (Mestrado em Oceanografia Biológica) - IO-USP, São Paulo. 43p.

ZARONI, L.P., ABESSA, D.M.S., LOTUFO, G.R., SOUSA, E.C.P.M. \& PINTO, Y.A. 2005. Testing with Embryos of Marine Mussels: Protocol Standardization for Perna perna (Linnaeus, 1758). Bull. Envir. Cont. Toxicol. 74:793-800. http://dx.doi. org/10.1007/s00128-005-0651-X 